\title{
Validation of quantitative fluorescent-PCR for rapid prenatal diagnosis of common aneuploidies in the Chinese population
}

\author{
A.-Q. Xu' ${ }^{1}$, M. Xia ${ }^{1}$, J.-T. Liu' ${ }^{2}$, F.-X. Yao ${ }^{3}$, W.-M. Zhang ${ }^{3}$, N. Hao ${ }^{2}$, J. Zhou ${ }^{2}$ \\ and X.-M. Bian ${ }^{2}$ \\ ${ }^{1}$ Department of Obstetrics and Gynecology, Affiliated Yuhuangding Hospital, \\ Qingdao University, Yantai, China \\ ${ }^{2}$ Department of Obstetrics and Gynecology, \\ Peking Union Medical College Hospital, \\ Chinese Academy of Medicine, Beijing, China \\ ${ }^{3}$ Department of Central Laboratory, Peking Union Medical College Hospital, \\ Chinese Academy of Medicine, Beijing, China \\ Corresponding author: X.-M. Bian \\ E-mail: xumingbian@163.com
}

Genet. Mol. Res. 12 (4): 6379-6388 (2013)

Received June 27, 2013

Accepted September 20, 2013

Published December 9, 2013

DOI http://dx.doi.org/10.4238/2013.December.9.1

\begin{abstract}
Quantitative fluorescent polymerase chain reaction (QF-PCR) is an accurate and reliable method for rapid detection of aneuploidy; however, it is not routinely used in China. We aimed to validate QF-PCR as a means for prenatal common aneuploidy screening and to analyze the heterozygosities of short tandem repeat (STR) markers in the Chinese population. The sequences of 19 STR markers in chromosomes $21,18,13, \mathrm{X}$, and $\mathrm{Y}$ were designed; three kinds of fluoresceins were used to label the primers, and the QFPCR detecting conditions were explored and optimized. The results of analysis of 210 prenatal samples by multiplex QF-PCR were compared with karyotyping analysis. All cases were successfully tested by QF-PCR and conventional cytogenetic analysis. QF-PCR
\end{abstract}


results were consistent with the results of cytogenetic analyses, with the exception of two cases. The sensitivity and specificity of QF-PCR to diagnose common aneuploidies were 94.74 and $100 \%$, respectively. The heterozygosities of most of the markers were lower than reported for Western populations, but relatively similar to those of other Asian populations. We conclude that QF-PCR is able to detect the common aneuploidies for prenatal diagnosis with high detection efficacy; therefore it is suitable for rapid prenatal diagnosis and for large-scale testing in laboratories. However, we need to add new STR markers or to find alternative STR markers with high heterozygosity in order to make this technique useful for routine diagnosis.

Key words: QF-PCR; Prenatal diagnosis; Aneuploidy

\section{INTRODUCTION}

Karyotyping is the gold standard in prenatal diagnosis. Chorionic villus samples (CVS), amniotic fluid, and fetal blood can be analyzed by this cytogenetic method. It can identify aneuploidy and unbalanced structural rearrangements in the fetus with high accuracy (Nicolini et al., 2004). However, the main disadvantage for karyotyping is the lengthy time it takes from sampling to getting the results. During this waiting period, patients often feel worried and anxious. In Europe, it takes 2 weeks to get the report, whereas in China most laboratories take more than 3 weeks to get the results.

Since the 1970s, great advances have been made to improve the efficiency of prenatal diagnoses, yet there are still many downsides and disadvantages to be addressed (Breuning, 2005; Shaffer and Bui, 2007). Due to the lack of efficient detection techniques, the demands for rapid aneuploidy diagnosis (RAD) are increasing in recent years. Among the wide spectrum of chromosomal abnormalities, 21, 18, 13, X and $\mathrm{Y}$ chromosomes are the most common sites where chromosome abnormalities occur among newborns. RAD targets abnormalities on the aforementioned chromosomes. The first technique for RAD is fluorescence in situ hybridization (FISH) (Klinger et al., 1992). Using FISH, rapid results may be obtained on interphase stage cells (Tepperberg et al., 2001; Leung et al., 2004; Caine et al., 2005; Wyandt et al., 2006). However, this technique is relatively expensive, and more labor-intensive (Cirigliano et al., 2002; Hulten et al., 2003; Donaghue et al., 2005; South et al., 2008).

Quantitative fluorescence polymerase chain reaction (QF-PCR) is an alternative choice for RAD. It was first introduced in rapid prenatal diagnosis in the early 1990s (Petersen et al., 1991; von Eggeling et al., 1993; Mansfield, 1993; Pertl et al., 1994). It is a rapid method for the detection of common aneuploidies by PCR amplicaion of short tandem repeats (STRs) in human chromosomes (Adinolfi et al., 1997; Cirigliano et al., 1999; Mann et al., 2001; Ogilvie et al., 2005). Compared with interphase FISH, the QFPCR technique has some advantages; in particular, it is more economic and automated and can be used in large scale detection (Hulten et al., 2003; El Mouatassim et al., 2004; Shaffer and Bui, 2007).

Currently, QF-PCR has been widely used in the most developed countries, but 
has not been used in China. Because STRs have shown polymorphisms and variations were observed in different populatons, some healthcare researchers think it needs further validation before its wide use in clinical settings (Cho et al., 2009). For example, Americans have lower heterozygosity at the locus D18S391 (0.75), whereas they have higher heterozygosity at the two loci P39 and DXS8377 (0.87 and 0.95, respectively) (Brown et al., 2006). But, in a Southeast Asian population, Quaife et al. (2004) reported that the heterozygosity for D18S391 was as low as 0.61 . Gole et al. (2008) reported that among the Asian ethnic groups, the Chinese showed a higher heterozygosity index for X22 (90\%) as compared to the Indians $(72.7 \%)$ and Malays $(66.6 \%)$. These authors also found that the three ethnic groups showed similar heterozygosity for the XHPRT marker, i.e., 69.3, 72.7 and $72.2 \%$ for Chinese, Indians and Malaysians, respectively. In another study, Cirigliano et al. (2009) reported that the Caucasian population showed a similar heterozygosity index for X22 (91\%) as the Chinese population, while a 75\% heterozygosity index for XHPRT, which was higher than that for the three Asian ethnic groups. Thus, the heterozygosity for the various STR loci needs to be determined before QF-PCR can be used in rapid prenatal diagnosis.

To establish the molecular basis for using RAD to detect the common aneuploidies in prenatal specimens, we performed a prospective study to validate QF-PCR. We used multiple QF-PCR to test 210 fetal samples, including amniotic fluid and chorionic villi. The heterozygosity for each STR locus was investigated. This is the first multiple QF-PCR study carried out in prenatal diagnosis in China.

\section{MATERIAL AND METHODS}

\section{Samples}

Samples were collected between May 2008 and September 2009. A total of 210 prenatal samples were included in this study. The majority of prenatal samples were amniotic fluids (133) collected between 16 and 22 weeks of gestation and CVS (39) collected between 11 and 14 weeks of gestation; 20 fetal blood samples and 18 villous from aborted fetuses were also investigated. Both QF-PCR and conventional cytogenetic studies were performed on all samples.

\section{Methods}

Genomic DNA was extracted from $2 \mathrm{~mL}$ amniotic fluid, $200 \mu \mathrm{L}$ fetal blood, and a small villous fragment using a QIAamp DNA Mini kit (Qiagen, Germany) according to the manufacturer instructions. The visible blood stained samples were excluded in this study. Six markers were used for chromosome 21, four for chromosomes 13 and 18, three for X chromosome and one for Y chromosome; the non-polymorphic sequence of the amelogenin (AMXY) gene was also included to determine fetal sex.

All forward primers were labeled with fluorescent molecules allowing for accurate sizing and quantification of QF-PCR products. Primers producing amplicons of similar sizes were labeled with different fluorochromes to be amplified and analyzed in the same multiplex QF-PCR.

In the first set of QF-PCR reactions, 8 markers were co-amplified in one multiplex QF-PCR assay, which included AMXY, DXS8377, D21S1435, IFNAR, D18S978, D18S535, 
D13S634, and D13S305. In the second set of QF-PCR reactions, another 8 markers were used, including DXS6803, DYS448, D21S11, D21S1270, D18S391, D18S386, D13S742, and D13S628. If definitive conclusions could not be drawn according to the results from these two sets of QFPCR, a third set of 3 markers would be used, namely P39, D21S1411 and D21S226.

QF-PCR was performed in $25 \mu \mathrm{L}$ containing genomic DNA, $200 \mu \mathrm{M}$ dNTPs, 1.6-22 pmol each primer (Sangon Biological Engineering Technology \& Services Co., Ltd.) (Tables 1, 2 and 3), $1.5 \mathrm{mM} \mathrm{MgCl}, 2$ U Taq polymerase and $1 \mathrm{X} \mathrm{Taq}$ polymerase buffer. Initial denaturation at $95^{\circ} \mathrm{C}$ for $5 \mathrm{~min}$ was followed by 25 cycles of $95^{\circ} \mathrm{C}$ for $25 \mathrm{~s}, 55^{\circ} \mathrm{C}$ for $45 \mathrm{~s}$, and $72^{\circ} \mathrm{C}$ for $30 \mathrm{~s}$. Final extension was for $20 \mathrm{~min}$ at $72^{\circ} \mathrm{C}$.

One microliter of PCR products was mixed with $10 \mu \mathrm{L}$ formamide and $0.5 \mu \mathrm{L}$ size standard in a MicroAmp (Applied Biosystems) optical 96-well reaction plate. After being denatured for $2 \mathrm{~min}$ at $95^{\circ} \mathrm{C}$ and cooled for $3 \mathrm{~min}$ at $-20^{\circ} \mathrm{C}$, this mix was run on an $\mathrm{ABI}$ model 3130 capillary electrophoresis instrument using POP-7 polymer. For fragment analysis, Genescan Analysis version 3.0 was used on a 3130 Genetic Analyzer.

Table 1. QF-PCR primer mix No. 1.

\begin{tabular}{|c|c|c|c|c|}
\hline Marker & Location & Size & Sequence and label & $\mathrm{pmol} /$ reaction \\
\hline \multirow[t]{2}{*}{ AMXY } & $\mathrm{Xp} 22.1-22.31$ & X 104 & FAM- CCCTGGGCTCTGTAAAGAATAGTG(F) & \multirow[t]{2}{*}{1.6} \\
\hline & Yp11.2 & Y 110 & ATCAGAGCTTAAACTGGGAAGCTG(R) & \\
\hline \multirow[t]{2}{*}{ D21S1435 } & $21 \mathrm{q} 21.3$ & $160-222$ & HEX- CCCTCTCAATTGTTTGTCTACC(F) & \multirow[t]{2}{*}{14} \\
\hline & & & ACAAAAGGAAAGCAAGAGATTTCA(R) & \\
\hline \multirow[t]{2}{*}{ D18S978 } & $18 \mathrm{q} 12.3$ & $180-220$ & TAMRA- GTAGATCTTGGGACTTGTCAGA(F) & \multirow[t]{2}{*}{10} \\
\hline & & & GTCTCCCATGGTCACAATGCT(R) & \\
\hline \multirow[t]{2}{*}{ DXS8377 } & $\mathrm{Xq} 28$ & $203-245$ & FAM- CACTTCATGGCTTACCACAG(F) & \multirow[t]{2}{*}{8} \\
\hline & & & GACCTTTGGAAAGCTAGTGT(R) & \\
\hline \multirow[t]{2}{*}{ IFNAR } & $21 \mathrm{q} 22.1$ & $370-410$ & FAM- CATTTGATCTTAGCCATCTATTGC(F) & \multirow{2}{*}{18} \\
\hline & & & ACTATGCAGCCATTTGAAAGACTA(R) & \\
\hline \multirow[t]{2}{*}{ D13S634 } & $13 q 14.3-q 22$ & $385-440$ & TAMRA- GGCAGATTCAATAGGATAAATAGA(F) & \multirow[t]{2}{*}{18} \\
\hline & & & GTAACCCCTCAGGTTCTCAAGTCT(R) & \\
\hline \multirow[t]{2}{*}{ D13S305 } & $13 q 12.1-q 14.1$ & $430-465$ & HEX- GCCTGTTTGAGGACCTGTCGTTA(F) & \multirow[t]{2}{*}{10} \\
\hline & & & TGGTTATAGAGCAGTTAAGGCAC(R) & \\
\hline \multirow[t]{2}{*}{ D18S535 } & $18 \mathrm{q} 12.3$ & $455-550$ & TAMRA- CAGCAAACTTCATGTGACAAAAGC(F) & \multirow[t]{2}{*}{20} \\
\hline & & & CAATGGTAACCTACTATTTACGTC(R) & \\
\hline
\end{tabular}

FAM, HEX and TAMRA are standard fluorescent dyes.

Table 2. QF-PCR primer mix No. 2.

\begin{tabular}{|c|c|c|c|c|}
\hline Marker & Location & Size & Sequence and label & $\mathrm{pmol} /$ reaction \\
\hline DXS6803 & $\mathrm{Xq12-q21.33}$ & $106-125$ & $\begin{array}{l}\text { HEX- GAAATGTGCTTTGACAGGAA(F) } \\
\text { CAAAAAGGGACATATGCTACTT(R) }\end{array}$ & 10 \\
\hline D18S391 & 18pter-p11.22 & $140-180$ & $\begin{array}{l}\text { FAM- GGACTTACCACAGGCAATGTGACT(F) } \\
\text { CTGGCTAATTGAGTTAGATTACAA(R) }\end{array}$ & 6 \\
\hline D21S11 & $21 \mathrm{q} 21.1$ & $225-280$ & $\begin{array}{l}\text { TAMRA- TTTCTCAGTCTCCATAAATATGTG(F) } \\
\text { GATGTTGTATTAGTCAATGTTCTC(R) }\end{array}$ & 16 \\
\hline D13S742 & $13 \mathrm{q} 11-\mathrm{q} 21.1$ & $235-315$ & $\begin{array}{l}\text { HEX-ATAACTGGGCTAGGAATGGAAATA(F) } \\
\text { GACTTCCCAATTCAGGAGGACT(R) }\end{array}$ & 8 \\
\hline D21S1270 & $21 \mathrm{q} 21-\mathrm{q} 22.1$ & $285-340$ & $\begin{array}{l}\text { TAMRA- CTATCCCACTGTATTATTCAGGGC(F) } \\
\text { TGAGTCTCCAGGTTGCAGGTGACA(R) }\end{array}$ & 22 \\
\hline D18S386 & $18 \mathrm{q} 22.1$ & $330-400$ & $\begin{array}{l}\text { FAM- TGAGTCAGGAGAATCACTTGGAAC(F) } \\
\text { CTCTTCCATGAAGTAGCTAAGCAG(R) }\end{array}$ & 12 \\
\hline DYS448 & Yq11.2 & $350-380$ & $\begin{array}{l}\text { TAMRA- CAAGGATCCAAATAAAGAACAGAGA(F) } \\
\text { GGTTATTTCTTGATTCCCTGTG(R) }\end{array}$ & 10 \\
\hline D13S628 & $13 q 31-q 32$ & $425-470$ & $\begin{array}{l}\text { FAM- TAACATTCATTGTCCCTTACAGAT(F) } \\
\text { GCAAGGCTATCTAACGATAATTCA(R) }\end{array}$ & 15 \\
\hline
\end{tabular}




\begin{tabular}{|c|c|c|c|c|}
\hline Marker & Location & Size & Sequence and label & $\mathrm{pmol} / \mathrm{reaction}$ \\
\hline P39 & $\mathrm{Xq} 28$ & $140-166$ & $\begin{array}{l}\text { FAM- AGCACATGGTATAATGAACCTCCACG(F) } \\
\text { CAGTGTGAGTAGCATGCTAGCATTTG(R) }\end{array}$ & 2 \\
\hline D21S1411 & $21 \mathrm{q} 22.3$ & $256-340$ & $\begin{array}{l}\text { FAM- ATAGGTAGATACATAAATATGATGÁ(F) } \\
\text { TATTAATGTGTGTCCTTCCAGGC(R) }\end{array}$ & 6 \\
\hline D21S226 & $21 \mathrm{q} 22.1$ & $440-470$ & $\begin{array}{l}\text { FAM- GCAAATTTGTGGATGGGATTAACAG (F) } \\
\text { AAGCTAAATGTCTGTAGTTATTCT(R) }\end{array}$ & 2 \\
\hline
\end{tabular}

\section{Interpretation of results}

For interpretation of results, the following criteria were used: allele dosage ratios (shorter allele/longer allele) between 0.8 and 1.4 were defined as normal, and an allele ratio greater than 1.8 , less than 0.65 , or the presence of three alleles of equal areas indicated trisomy. A single peak was described as uninformative. A minimum of two informative markers for each chromosome $(21,18,13$, and X) was required for confident interpretations.

\section{RESULTS}

\section{Comparison with conventional cytogenetic analysis}

All cases were successfully tested by QF-PCR and conventional cytogenetic analysis. The results of these two methods are presented in Table 4. Eight cases of trisomy 21, 3 cases of trisomy 18, 2 cases of triploidy, 1 case of Turner's syndrome, 2 cases of Klinefelter's syndrome, 1 case of 47,XXX, and 1 case of 47,XYY were detected by QF-PCR (Figures 1-4). There were no false positives. QF-PCR results were consistent with the results of cytogenetic analyses, with the exception of two cases. The undetected cases were an inherited balanced structural abnormality with karyotype $46, X X, t(11 ; 20)$. Another case of $46, X X$ produced an uninformative result for all the markers of X chromosome by QF-PCR, and it was confirmed by interphase FISH.

\section{Table 4. Results of testing 210 fetal samples by QF-PCR and conventional cytogenetic analysis.}

\begin{tabular}{lcc}
\hline Karytotype & Cytogenetics & QF-PCR \\
\hline $46, \mathrm{XX} ; 46, \mathrm{XY}$ & 191 & 192 \\
47,XX +21;47,XY+21 & 8 & 8 \\
47,XX +18;47,XY+18 & 3 & 3 \\
69,XXX & 2 & 2 \\
45,X & 1 & 1 \\
47,XXY & 2 & 1 \\
47,XXX & 1 & 1 \\
47,XYY & 1 & 0 \\
Structural balanced & 1 & 94.74 \\
Total abnormalities & 19 & 100 \\
Sensitivity (\%) & & 100 \\
Specificity (\%) & & 99.48 \\
Positive predictive value (\%) & & \\
Negative predictive value (\%) & & \\
\hline
\end{tabular}






Figure 1. Genotype profiles of a normal diploid sample amplified with the multiplex QF-PCR and analyzed on a 3130 Genetic Analyzer. Fragment size in base pairs shown on the horizontal axis, arbitrary fluorescence units on the vertical axis. A. First set of QF-PCR: 1) AMXY (blue), 2) D21S1435 (green), 3) D18S978 (red), 4) DXS8377 (blue), 5) IFNAR (blue), 6) D13S634 (red), 7) D13S305 (green), 8) D18S535 (red); B. Second set of QF-PCR: 1) DXS6803 (green), 2) D18S391 (blue), 3) D21S11 (red), 4) D13S742 (green), 5) D21S1270 (red), 6) D18S386 (blue), 7) DYS448 (red), 8) D13S628 (blue). All markers are heterozygous and exhibit 2 alleles with peak areas in a 1:1 ratio except DXS8377, DXS6803, D13S742, and DYS448, which are homozygous. The karyotyping result are $46, X Y$.

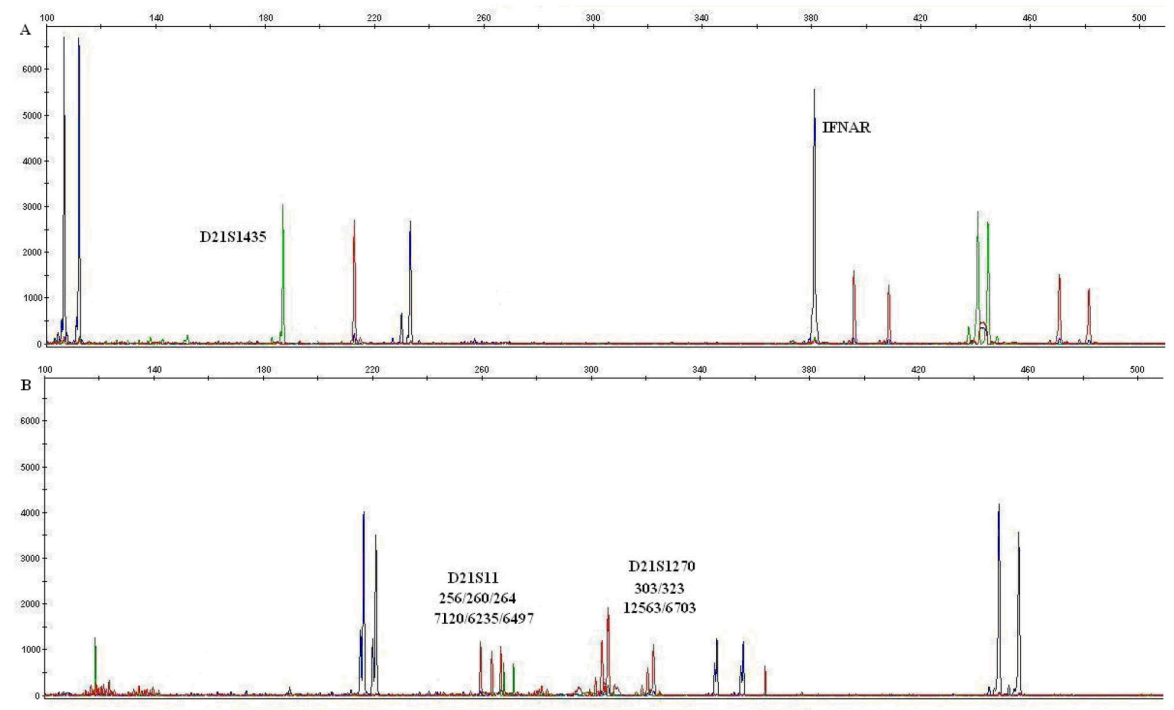

Figure 2. Genotype profiles of a trisomy 21 sample amplified with the multiplex QF-PCR and analyzed on a 3130 Genetic Analyzer. Chromosome 21 STR markers exhibit 3 (D21S11) or 2 alleles in 2:1 peak ratio (D21S1270); D21S1435 and IFNAR are homozygous and therefore uninformative. The karyotyping result is 47,XY+21. 


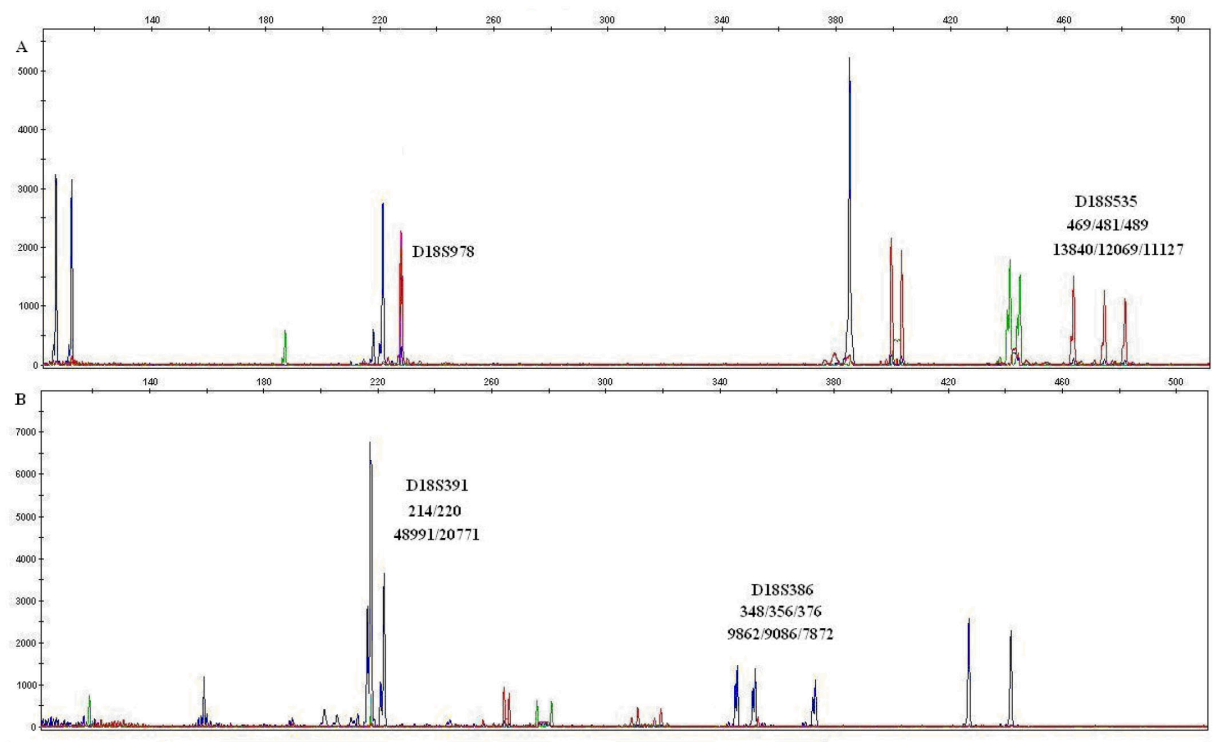

Figure 3. Genotype profiles of a trisomy 18 sample amplified with the multiplex QF-PCR and analyzed on a 3130 Genetic Analyzer. Chromosome 18 STR markers exhibit 3 (D18S535 and D18S386) or 2 alleles in 2:1 peak ratio (D18S391); D18S978 is homozygous and therefore uninformative. The karyotyping result is $47, \mathrm{XY}+18$.

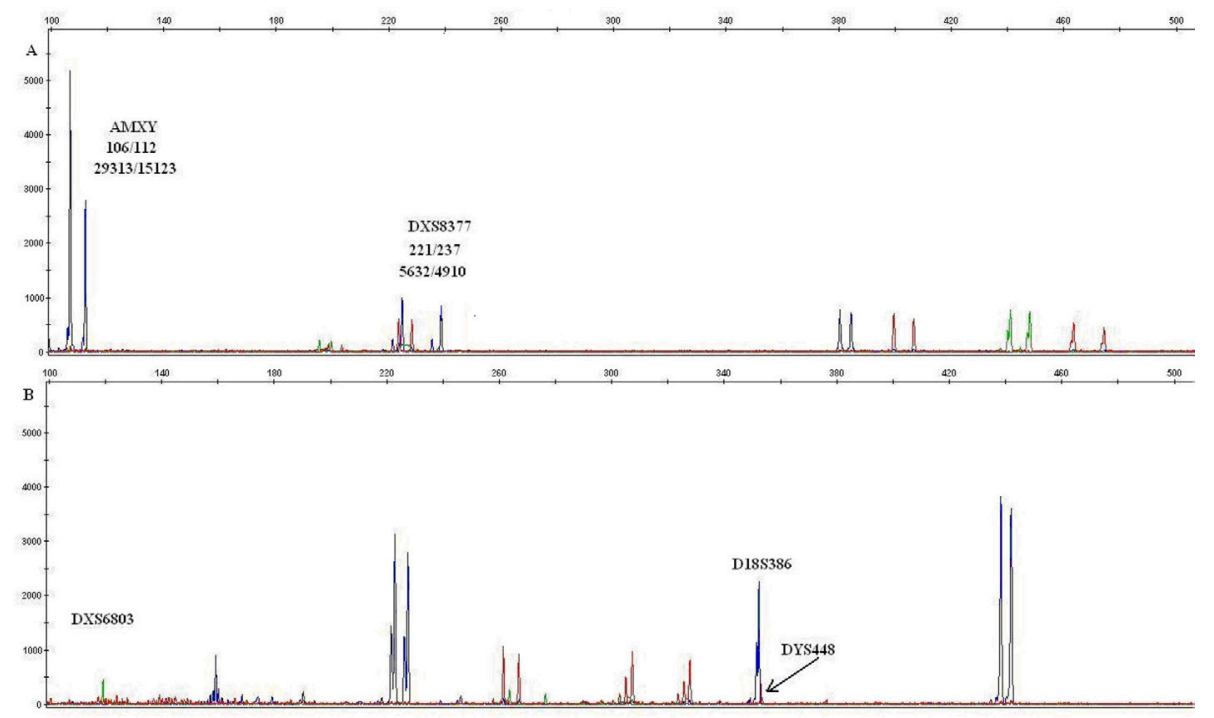

Figure 4. Genotype profiles of a Klinefelter's syndrome sample amplified with the multiplex QF-PCR and analyzed on a 3130 Genetic Analyzer. Sex chromosome STR markers exhibit 2 alleles in 2:1 peak ratio (AMXY); DXS8377, DXS6803 and DYS448 are homozygous. Chromosome 13, 18 and 21 STR markers exhibit 2 alleles with a normal 1:1 peak ratio except D18S386. The karyotyping result is $47, \mathrm{XXY}$. 


\section{Analysis of the heterozygosities of STR markers}

The heterozygosities of markers selected for QF-PCR are given in Table 5. We compared our results of STR markers in the Chinese population with Western populations (Cirigliano et al., 2004) and other Asian populations (Cho et al., 2009). The heterozygosities of all markers in chromosome 21 were markedly lower than in Western populations, but relatively similar with other Asian populations. The heterozygosities for chromosome 18 from our study samples were lower than in Western populations, with the exception of D18S535, in which heterozygosity was higher than in the Korean population and Southeast Asian population. Among the markers of chromosome 13, D13S634 showed a lower heterozygosity than in Western populations and other Asian populations, while D13S305 was higher than in Western populations. As for the sex chromosome, DXS6803 and DXS8377 showed higher a heterozygosity in Western populations than Chinese populations.

Table 5. List of STR markers analyzed in Chinese population.

\begin{tabular}{lccl}
\hline Marker & Heterozygosities & Allele No. & Allele length $(\mathrm{bp})$ \\
\hline D21S1435 & 0.70 & 8 & $174,178,182,186,190,194,198,202$ \\
IFNAR & 0.65 & 7 & $376,380,384,388,392,396,400$ \\
D21S226 & 0.46 & 6 & $442,446,450,454,458,462$ \\
D21S1411 & 0.79 & 13 & $260,276,284,292,296,300,304,308,312,316,320,324,328$ \\
D21S1270 & 0.80 & 17 & $275,279,287,291,295,299,303,307,311,315,319,323,327,331,335,347,355$ \\
D21S11 & 0.75 & 8 & $232,248,252,256,260,264,268,272$ \\
D18S535 & 0.84 & 14 & $437,445,449,453,457,461,465,469,473,477,481,485,489,493$ \\
D18S391 & 0.63 & 13 & $154,158,174,178,190,206,210,214,218,222,226,230,234$ \\
D18S386 & 0.82 & 13 & $340,344,348,352,356,360,364,368,372,376,380,384,388$ \\
D18S978 & 0.82 & 10 & $199,203,207,211,215,219,223,227,231,239$ \\
D13S742 & 0.77 & 16 & $248,252,256,260,264,268,272,276,280,284,288,292,300,304,308,320$ \\
D13S305 & 0.77 & 11 & $380,396,404,424,428,432,436,440,444,448,452,456,460$ \\
D13S634 & 0.71 & 10 & $383,391,395,399,403,407,408,411,415,419,423$ \\
D13S628 & 0.66 & 13 & $423,427,431,435,439,443,447,451,455,459$ \\
DXS8377 & 0.79 & 7 & $213,217,221,225,229,233,237,241,245,249,253,257,261$ \\
DXS6803 & 0.52 & 4 & $102,106,110,114,118,122,126$ \\
P39 & 0.50 & & $146,154,158,162$ \\
DYS448 & & & $X: 104-106 ; Y: 110-112$ \\
AMXY & - & &
\end{tabular}

\section{DISCUSSION}

QF-PCR has been applied in rapid aneuploidy diagnosis for almost twenty years. This technique has been improved and widely used nowadays. It has many advantages, such as accurate, easy to manipulate, quick to generate results, high throughput to test samples, cost effective, etc. In this study, we adapted a single-tube QF-PCR technique in which 8 markers (2 STR markers specific for each chromosome 13, 18, 21 or sex chromosome) were co-amplified in one multiplex PCR assay. Apart from one specimen of inherited balanced structural abnormality with karyotype 46,XX,t(11;20), all the aneuploid abnormalities involving chromosomes 13, $18,21, \mathrm{X}$ and $\mathrm{Y}$ were detected, and there was no discordance between the results with QF-PCR and conventional cytogenetics. However, one of the main disadvantages for QF-PCR is that it cannot easily differentiate 46,XX from 45,XO (Cirigliano et al., 2002). Therefore, interphase FISH should be considered in detecting 45,XO. In this study, one case with 46,XX gave an uninformative result for X chromosome because all the STR markers in X chromosome showed 
a single peak which meant to be homozygous. We then performed interphase FISH and confirmed 46,XX. Another case with 46,XX,t(11;20) belonged to paternal balanced translocation. This abnormality is beyond the five chromosomes that QF-PCR was designed to target, and therefore, this case was not considered a misdiagnosis (Mann et al., 2004).

As Brown suggested, each laboratory that uses QF-PCR needs to perform an independent validation test. This will demonstrate the heterozygosities of various STR loci and the efficiency of multiplex combinations. We have done extensive literature reviews for the choice of the STR markers, and selected the most popular STR markers involving 13, 18, $21, \mathrm{X}$ and $\mathrm{Y}$ chromosome to be used in our laboratory. From this study, we found that the heterozygosities of most of STR markers in Chinese populations were lower than those in Western populations, where especially all markers in chromosome 21 were markedly lower than in Western populations. The reasonable explanation for this phenomenon may be that the Chinese population was more conservative culturally and historically, and marriage between different ethnic groups is not commonly seen (Quaife et al., 2004). From this study, the STR markers for chromosome 21 show lower heterozygosities, so we need to add new STR markers or alternative STR markers with high heterozygosity in the future studies. In addition, we

plan to choose new markers in X chromosome or autosomal markers as internal control for quantification. The data from this assay help assessing the feasibility of applying QF-PCR kits to Chinese populations.

\section{ACKNOWLEDGMENTS}

We thank Dr. Han Juanjuan and Dr. Su Yao for experimental support, and Dr. Yu Hong for biostatistical support.

\section{REFERENCES}

Adinolfi M, Pertl B and Sherlock J (1997). Rapid detection of aneuploidies by microsatellite and the quantitative fluorescent polymerase chain reaction. Prenat. Diagn. 17: 1299-1311.

Breuning MH (2005). Genetic testing: from chromosomes to DNA, a revolution in prenatal diagnosis. Eur. J. Hum. Genet. 13: 517-518.

Brown L, Abigania M, Warburton D and Brown S (2006). Validation of QF-PCR for prenatal aneuploidy screening in the United States. Prenat. Diagn. 26: 1068-1074.

Caine A, Maltby AE, Parkin CA, Waters JJ, et al. (2005). Prenatal detection of Down's syndrome by rapid aneuploidy testing for chromosomes 13, 18, and 21 by FISH or PCR without a full karyotype: a cytogenetic risk assessment. Lancet 366: 123-128.

Cho EH, Park BY, Kang YS and Lee EH (2009). Validation of QF-PCR in a Korean population. Prenat. Diagn. 29: 213-216.

Cirigliano V, Sherlock J, Conway G, Quilter C, et al. (1999). Rapid detection of chromosomes X and Y aneuploidies by quantitative fluorescent PCR. Prenat. Diagn. 19: 1099-1103.

Cirigliano V, Ejarque M, Fuster C and Adinolfi M (2002). X chromosome dosage by quantitative fluorescent PCR and rapid prenatal diagnosis of sex chromosome aneuploidies. Mol. Hum. Reprod. 8: 1042-1045.

Cirigliano V, Voglino G, Canadas MP, Marongiu A, et al. (2004). Rapid prenatal diagnosis of common chromosome aneuploidies by QF-PCR. Assessment on 18,000 consecutive clinical samples. Mol. Hum. Reprod. 10: 839-846.

Cirigliano V, Voglino G, Ordonez E, Marongiu A, et al. (2009). Rapid prenatal diagnosis of common chromosome aneuploidies by QF-PCR, results of 9 years of clinical experience. Prenat. Diagn. 29: 40-49.

Donaghue C, Mann K, Docherty Z and Ogilvie CM (2005). Detection of mosaicism for primary trisomies in prenatal samples by QF-PCR and karyotype analysis. Prenat. Diagn. 25: 65-72.

El Mouatassim S, Becker M, Kuzio S, Ronsin C, et al. (2004). Prenatal diagnosis of common aneuploidies using multiplex quantitative fluorescent polymerase chain reaction. Fetal Diagn. Ther. 19: 496-503. 
Gole L, Adrianne F, Ee AM, Ng BL, et al. (2008). Refining quantitative fluorescent polymerase chain reaction for prenatal detection of X chromosomal anomalies in the major Southeast Asian populations. Singapore Med. J. 49: 1025-1028.

Hulten MA, Dhanjal S and Pertl B (2003). Rapid and simple prenatal diagnosis of common chromosome disorders: advantages and disadvantages of the molecular methods FISH and QF-PCR. Reproduction 126: 279-297.

Klinger K, Landes G, Shook D, Harvey R, et al. (1992). Rapid detection of chromosome aneuploidies in uncultured amniocytes by using fluorescence in situ hybridization (FISH). Am. J. Hum. Genet. 51: 55-65.

Leung WC, Lau ET, Lao TT and Tang MH (2004). Rapid aneuploidy screening (FISH or QF-PCR): the changing scene in prenatal diagnosis? Expert Rev. Mol. Diagn. 4: 333-337.

Mann K, Fox SP, Abbs SJ, Yau SC, et al. (2001). Development and implementation of a new rapid aneuploidy diagnostic service within the UK National Health Service and implications for the future of prenatal diagnosis. Lancet 358: 1057-1061.

Mann K, Donaghue C, Fox SP, Docherty Z, et al. (2004). Strategies for the rapid prenatal diagnosis of chromosome aneuploidy. Eur. J. Hum. Genet. 12: 907-915.

Mansfield ES (1993). Diagnosis of Down syndrome and other aneuploidies using quantitative polymerase chain reaction and small tandem repeat polymorphisms. Hum. Mol. Genet. 2: 43-50.

Nicolini U, Lalatta F, Natacci F, Curcio C, et al. (2004). The introduction of QF-PCR in prenatal diagnosis of fetal aneuploidies: time for reconsideration. Hum. Reprod. Update 10: 541-548.

Ogilvie CM, Donaghue C, Fox SP, Docherty Z, et al. (2005). Rapid prenatal diagnosis of aneuploidy using quantitative fluorescence-PCR (QF-PCR). J. Histochem. Cytochem. 53: 285-288.

Pertl B, Yau SC, Sherlock J, Davies AF, et al. (1994). Rapid molecular method for prenatal detection of Down's syndrome. Lancet 343: 1197-1198.

Petersen MB, Schinzel AA, Binkert F, Tranebjaerg L, et al. (1991). Use of short sequence repeat DNA polymorphisms after PCR amplification to detect the parental origin of the additional chromosome 21 in Down syndrome. Am. J. Hum. Genet. 48: 65-71.

Quaife R, Wong LF, Tan SY, Chua WY, et al. (2004). QF-PCR-based prenatal detection of aneuploidy in a southeast Asian population. Prenat. Diagn. 24: 407-413.

Shaffer LG and Bui TH (2007). Molecular cytogenetic and rapid aneuploidy detection methods in prenatal diagnosis. Am. J. Med. Genet. C Semin. Med. Genet. 145C: 87-98.

South ST, Chen Z and Brothman AR (2008). Genomic medicine in prenatal diagnosis. Clin. Obstet. Gynecol. 51: 62-73.

Tepperberg J, Pettenati MJ, Rao PN, Lese CM, et al. (2001). Prenatal diagnosis using interphase fluorescence in situ hybridization (FISH): 2-year multi-center retrospective study and review of the literature. Prenat. Diagn. 21: 293301.

von Eggeling F, Freytag M, Fahsold R, Horsthemke B, et al. (1993). Rapid detection of trisomy 21 by quantitative PCR. Hum. Genet. 91: 567-570.

Wyandt HE, Tonk VS, Huang XL, Evans AT, et al. (2006). Correlation of abnormal rapid FISH and chromosome results from amniocytes for prenatal diagnosis. Fetal Diagn. Ther. 21: 235-240. 